\title{
The Sloan Bright Arcs Survey: Four Strongly Lensed Galaxies with Redshift $>2$
}

\author{
H. Thomas Diehl ${ }^{1}$, Sahar S. Allam ${ }^{1}$, James Annis ${ }^{1}$, Elizabeth J. Buckley-Geer ${ }^{1}$, \\ Joshua A. Frieman ${ }^{1,2}$, Donna Kubik ${ }^{1}$, Jeffrey M. Kubo ${ }^{1}$, Huan Lin $^{1}$, Douglas Tucker ${ }^{1}$, \\ Anderson West ${ }^{1,3}$
}

\begin{abstract}
We report the discovery of four very bright, strongly-lensed galaxies found via systematic searches for arcs in Sloan Digital Sky Survey Data Release 5 and 6. These were followed-up with spectroscopy and imaging data from the Astrophysical Research Consortium 3.5m telescope at Apache Point Observatory and found to have redshift $z>2.0$. With isophotal magnitudes $r=19.2-20.4$ and $3^{\prime \prime}$-diameter magnitudes $r=20.0-20.6$, these systems are some of the brightest and highest surface brightness lensed galaxies known in this redshift range. In addition to the magnitudes and redshifts, we present estimates of the Einstein radii, which range from $5.0^{\prime \prime}$ to $12.7^{\prime \prime}$, and use those to derive the enclosed masses of the lensing galaxies.
\end{abstract}

Subject headings: gravitational lensing — galaxies: high-redshift

\section{Introduction}

Strong gravitational lenses provide an opportunity for studying properties of distant galaxies. Because surface brightness is unchanged during lensing, magnification of the image provides amplification of the image flux and allows studies of details that would otherwise be too faint for detailed ground-based investigation. Models of the lens systems also provide information on the mass distribution, including dark matter, of the lenses themselves.

Recent wide area optical surveys have enabled identification of new samples of strong lens systems. The Sloan Digital Sky Survey (SDSS) (York et al. 2000) spectroscopic data

\footnotetext{
${ }^{1}$ Fermi National Accelerator Laboratory, P.O. Box 500, Batavia, IL 60510

${ }^{2}$ Department of Astronomy and Astrophysics, University of Chicago, 5640 South Ellis Avenue, Chicago, IL 60637

${ }^{3}$ The Illinois Math and Science Academy, Aurora, IL 60506
} 
has been used (Bolton et al. 2008; Willis et al. 2006) to identify $\sim 140$ strong lensing candidates in systems where multiple spectra appear in a single $3^{\prime \prime}$-wide spectral fiber. The SDSS imaging data provides the opportunity to identify a complementary set of strong lens systems that have a larger lens-arc separation $\left(>3.0^{\prime \prime}\right)$. An imaging search of 240 rich clusters (Hennawi et al. 2008) yielded 16 strong lens systems with large $\left(>10^{\prime \prime}\right)$ radius, where the lensing interpretation is based on morphology, and 21 additional systems for which the lensing interpretation is less certain. Several more large arcs in the SDSS data have been described in recent papers (Belokurov et al. 2009; Wen et al. 2009). The CFHTLS field and COSMOS field have yielded 40 (Cabanac et al. 2007) and 70 (Faure 2008; Jackson 2008) strong lens candidate systems.

Relatively few arc systems have been reported where the lensed galaxy is known to have redshift $z>2$. Some systems with high surface brightness have been reported. The brightest Lyman Break Galaxy (LBG) currently known is SDSS J002240.91+143110.4 "The 8 o'clock arc" (Allam et al. 2007) with a redshift $z=2.73$ and magnitude $r=19.2$. Other bright systems include SDSS J120602.09+514229.5 "The Clone" (Lin et al. 2009) with redshift $z=2.0$ and magnitude $r=19.8$, MS 1512-cB58 (Yee et al. 1996) with redshift $z=2.73$ and magnitude $r=20.4$, and the system (Smail et al. 2007) lensed by MACS J2135.2-0102 with redshift $z=3.07$.

Motivated by our discovery of "The 8 o'clock arc", we initiated a program, the Sloan Bright Arcs Survey, to identify and confirm lensed high-redshift galaxies. We recently reported (Kubo et al. 2009) the discovery of six spectroscopically confirmed systems with redshifts $z=0.4$ to 1.4 . In this paper we report the discovery and confirmation of four more systems with redshift $z>2$.

This paper is organized as follows. In $\S 2$ we describe the strong-lens arc search samples and candidate identification procedure. In $\S 3$ we describe our follow-up imaging and spectroscopy procedure. In $\S 4$ we describe the results from the follow-up and use those results to calculate properties of the lensed galaxies. Finally, in $\S 5$, we describe further follow-up measurements underway and summarize.

\section{Arc Search Samples}

Between 2000 and 2008, the Sloan Digital Sky Survey imaged more than 8,400 square degrees of the extragalactic sky in $u, g, r, i$, and $z$-band filters and obtained spectra of 930,000

galaxies. We searched (Kubik 2007) for strong-gravitational lens galaxies in the SDSS Data Release 5 catalog (Adelman-McCarthy et al. 2007) around luminous red galaxies (LRG's) 
(Eisenstein et al. 2001) and brightest cluster galaxies (BCG's) (Hansen et al. 2005). These were sorted according to the number, $N$, of additional galaxies located within $10^{\prime \prime}$ that have a color $g-r<1$ and $r-i<1$. The list of candidates was provided to a web-based display tool that allows one to extract small false-color images using the SDSS Catalog Archive Server (CAS), based on a user-defined list of positions. We inspected the images to find ones where there was a spatially continuous arc or ring, or a nearly spatially continuous set of knots, (the source candidate) that is centered or nearly centered on a galaxy or galaxy cluster (the lens candidate). We required that the source candidate not have a distinct color-change over its length (to be consistent with a single lensed source interpretation) and that the source candidate not be continuously-connected to the central object by spiral arms, bars, or non-structural lumination. This was to eliminate galaxies that are providing both the central structure and the arc, ring, or knot. Independent inspections were performed by four different people and any candidate that was noted by two or more scanners was marked for further study. There were 14 such candidates.

In addition, a second list of candidates resulted from an independent inspection of an SDSS DR6 catalog of merging galaxies. In this catalog, a merging galaxy pair is defined (Allam et al. 2004) as two galaxies in the magnitude range $16.0<g<21.0$ separated by less than the sum of their respective Petrosian radii (Stoughton et al. 2002). There are many systems with morphology and color similar to our other strong lens candidates.

\section{Follow-up Imaging and Spectroscopy}

We followed up the 14 candidates from the first list and a subset of the candidates from the second list using the Astrophysical Research Consortium (ARC) 3.5m telescope at the Apache Point Observatory. Visible-wavelength imaging was performed using the SPICAM CCD imager, which has a field-of-view of 4.78 arcminutes square and a plate scale of $0.28^{\prime \prime}$ per pixel. We observed each candidate system in the three SDSS filters gri, taking in each filter typically three 300-second exposures, dithered with $15^{\prime \prime}$ offsets. The images were bias subtracted and flatfielded using standard IRAF tasks and were then coadded using the SWarp package (Bertin 2006). Astrometric calibration was carried out using matches to objects in the SDSS. Likewise, photometric calibration was done by matching to stars in the SDSS. Specifically, we used GALFIT (Peng et al. 2002) to fit Moffat profiles to stars in the images, and set the photometric zeropoint of an image by taking the median offset between the Moffat profile total magnitudes and the SDSS model magnitudes for these stars. Additional details of our reduction procedure may be found in reference (Lin et al. 2009).

We report magnitudes in $3^{\prime \prime}$-diameter circular apertures for our arc systems (except one 
instance as noted), where the photometry was done using SExtractor (Bertin \& Arnouts 1996 ) in dual-image mode, using the $r$-band image for each system to define object detection. Note that due to imperfect SExtractor deblending of the contaminating light of the lensing galaxies from that of the arcs, the magnitudes we quote for the arc components may be systematically too bright by a few tenths of a magnitude, especially in the $i$ and $r$ images where the red lensing galaxies are more prominent. We will defer to future analyses more careful photometric modeling of these systems (i.e., using GALFIT to subtract the lensing galaxy light, as in Lin et al. 2009) as a prelude to detailed lens modeling, but in the present discovery paper simple aperture magnitudes will suffice as reasonable first approximations to the photometry of the arc components. We also report SExtractor isophotal magnitudes for our arcs, where the limiting isophotes are the SExtractor $r$-band detection thresholds (1.5 times the image RMS). Unless specifically noted, all magnitudes reported are those from $3^{\prime \prime}$-diameter apertures.

Spectroscopy was obtained using the Dual-Imaging Spectograph (DIS III), a mediumdispersion spectrograph with separate red/blue grating setups. The spectral range is 3000 - $9600 \AA$ with a resolution of $1.83 \AA$ per pixel in the blue and $2.31 \AA$ per pixel in the red. Bright arcs and central galaxies were targeted, typically with three 900 second exposures using a $1.5^{\prime \prime}$ or $2.0^{\prime \prime}$ wide slit. The spectra were reduced and extracted using standard IRAF routines and were flux calibrated using observations of the spectrophotometric standard G191-B2B. Redshift measurements were done using the IRAF external task XCSAO, by cross correlation against the Lyman break galaxy (LBG) template of Shapley et al. (2003). The cross correlation was limited to the observed wavelength range $3500-5500 \AA$ (except for one system, as noted below), where all the relevant LBG spectroscopic features were located for our systems. Note we calibrated all our spectra to air wavelengths, so that the LBG template had to be transformed from vacuum to air wavelengths before being used for cross correlation. Dates for useful follow-up observations are listed in Tables 1 and 2.

\section{Lensing Systems}

We report results on four systems that were found to have source redshifts $z>2.0$. Figures 1 and 2 show color-coadd images of the four systems as observed from the ARC 3.5m telescope using SPICAM. Figures 3 - 6 show the spectra of the source arcs from the ARC 3.5m using DIS III. Table 3 provides the positions, redshifts and magnitudes of lensed images of the source galaxy. The Einstein radius $\left(\Theta_{E}\right)$ is estimated from a simple manual fit of a chord of a circle to the arc. Using $\Theta_{E}$ and the spectroscopically measured source and lens redshifts, we can estimate the mass enclosed within the Einstein radius 
$\left(M\left(<\Theta_{E}\right)\right)$. Here, a singular isothermal sphere (SIS) was used to approximate the lenses. This model is typically used for cluster-scale masses (Narayan and Bartelmann 1996). A flat cosmology with $\Omega_{\Lambda}=0.7, \Omega_{M}=0.3$, and $H_{0}=70 \mathrm{~km} / \mathrm{s} / \mathrm{Mpc}(h=0.7)$ is assumed for these calculations. These results are summarized in Table 4.

\subsection{SDSS J090122.37+181432.3}

SDSS J090122.37+181432.3 was selected based on the fact that it has three additional galaxies located within $10^{\prime \prime}$ of the central LRG that have a color $g-r<1$ and $r-i<1(N=3)$. Fig. 1 (left) shows the $g, r, i$ false color image of the system. There is a bright central LRG (SDSS J090122.37+181432.3) with many galaxies in the same cluster located nearby. The SDSS DR7 data provides the spectroscopic redshift $z=0.3459 \pm 0.0003$ of the central LRG.

The source is comprised of a long arc to the east of the central LRG. DIS spectroscopy provides the redshifts of the arc components to the northeast and southeast of the central LRG. The redshifts are consistent with the interpretation the arc is a single system. The mean redshift of the two components was $z=2.2558 \pm 0.0003$. The uncertainty on the redshift comes from the full spread of the measurements in slightly different setups of the slit. Fig. 3 shows that the spectra of the two components exhibit clear and strong emission, which we identify as Ly $\alpha$. [OII] 3727 at low redshift is ruled out by the absence of expected accompanying strong emission lines from [OIII] 5007 and in particular H $\alpha$. The Ly $\alpha$ identification and hence the redshift are also confirmed by clear detections of rest-frame

optical emission lines from near-IR spectroscopic observations (Hainline et al. 2009). The figure also marks the expected positions of typical LBG absorption features in the rest-frame UV (Shapley et al. 2003), but these lines are at best only weakly detected in the spectrum of knot b (maybe SiIV and CIV in the upper panel).

The Einstein radius of the arc, is $7.7 \pm 1.1^{\prime \prime}$. The uncertainty is a result of the change in curvature of the arc over its length. The mass within the Einstein radius is $9.6 \pm 2.7 \times 10^{12}$ $\mathrm{M}_{\odot}$. The uncertainty in the mass is dominated by the contribution from the uncertainty in the Einstein radius. That is the case for all of the systems described in this paper.

\subsection{SDSS J134332.85+415503.4}

SDSS J134332.85+415503.4 comes from the merging galaxy sample. Fig. 1 (right) shows the false color image of the system, which is comprised of a rich galaxy cluster with a bright central LRG. The SDSS catalog provides the spectroscopic redshift of two 
central galaxies that are within $10^{\prime \prime}$ of the lensed galaxy. The bright, central galaxy (SDSS $\mathrm{J} 134332.85+415503.4$ ) has redshift $z=0.4180 \pm 0.0002$. The second-brightest galaxy, located immediately southeast of the central LRG, has redshift $z=0.4135 \pm 0.0004$.

The arc, approximately $20^{\prime \prime}$ in length, is to the east and northeast of the central LRG. DIS spectroscopy of the arc provides $z=2.0927 \pm 0.0003$, where the uncertainty is based on the measured differences of the redshifts of knots in other systems. The spectrum is shown in Fig. 4. For comparison, this figure also shows, shifted to the same redshift, the spectrum of the composite LBG template (Shapley et al. 2003) that we are using for cross correlation and redshift measurement. Note the clear presence in the arc spectrum of all the marked typical LBG absorption features that are seen in the template. However, also note that the arc spectrum shows Ly $\alpha$ absorption instead of emission as in the template, and for this system (only) we have limited the cross correlation wavelength range to $3800-5500 \AA$ in order to avoid the Ly $\alpha$ emission line in the LBG template.

A simple fit determined that the Einstein radius is $12.7 \pm 0.4^{\prime \prime}$. The mass within the Einstein radius is $31.7 \pm 2.0 \times 10^{12} \mathrm{M}_{\odot}$.

\subsection{SDSS J114833.14+193003.2}

SDSS J114833.14+193003.2, a.k.a. "The Cosmic Horseshoe" (Belokurov et al. 2009), was selected from the $N=3$ sample in our search around LRGs. Fig. 2 (left) shows the false color image. The SDSS DR7 provides spectroscopic redshift $z=0.4457 \pm 0.0003$ for the central bright galaxy (SDSS J114833.14+193003.2). We emphasize that we identified and confirmed this as a strong-lensing system prior to our knowledge that another group was studying it.

The arc forms a nearly continuous ring around a central LRG. DIS spectroscopy provides an average redshift $z=2.3811 \pm 0.0003$ for the three bright knots arrayed around the central LRG. Again, the uncertainty is due to differences between individual redshifts of the knots. The spectra are shown in Fig. 5, where we see strong Ly $\alpha$ emission and the presence of most of the marked typical LBG absorption features.

A simple fit determined that the Einstein radius is $5.0 \pm 0.3^{\prime \prime}$. The mass within the Einstein radius is $5.1 \pm 0.6 \times 10^{12} \mathrm{M}_{\odot}$. The Einstein radius and enclosed mass are in agreement with those previously published (Belokurov et al. 2009). 


\subsection{SDSS J090002.79+223403.6}

SDSS J090002.79+223403.6 was selected from the $N=4$ sample in our search around LRGs. Fig. 2 (right) shows the false color image of the system, which has two bright central galaxies. The SDSS data provides the spectroscopic redshift, $z=0.4890 \pm 0.0002$, for the more northwest of those two galaxies.

The source galaxy appears as a knot to the east and an arc to the southeast of the lens and as a knot immediately to the west of the lens. Analysis of the DIS spectra, shown in Fig. 6, indicates that the redshifts of the knots to the northeast and northwest of the central LRG pair are the same within uncertainties. The mean is $z=2.0325 \pm 0.0003$. Again, we take an uncertainty based on differences between redshifts of knots in the other systems described in this paper. The spectra again show strong Ly $\alpha$ emission corroborated by detection of some of the typical LBG absorption features, in particular the pair of SiIV lines and the CIV line.

The simple fit determined that the Einstein radius is $7.0 \pm 0.8^{\prime \prime}$. The mass within the Einstein radius is $11.6 \pm 2.7 \times 10^{12} \mathrm{M}_{\odot}$.

\section{Summary and Conclusion}

Our strong lens search has yielded four systems with lensed galaxies that have redshift $z>2.0$. The lens redshifts are presented, based on the SDSS spectroscopic data. We measured the source galaxy redshifts using the ARC 3.5m DIS spectrograph. Further imaging using the ARC $3.5 \mathrm{~m}$ imager SPICAM revealed more detail about the lensed systems. The arcs from the lensed galaxies have Einstein radius between 5.0" and $12.7^{\prime \prime}$ and are quite bright. A simple SIS model was used to provide estimates of the mass enclosed within the Einstein radius of the lensing galaxies.

From the data in Table 3, we see that these systems have approximate total isophotal magnitudes $r=19.2-20.4$. For comparison with other lensed systems, we use the $3^{\prime \prime}$ diameter magnitudes and those are $r=20.0-20.6$. Thus these systems are about one magnitude fainter than the brightest lensed LBG known, the 8 o'clock arc $(r=19.2$ ) (Allam et al. 2007), but are quite similar to the few other very bright $z>2$ lensed systems, such as MS 1512cB58 $(r=20.4)$ (Yee et al. 1996) and the Clone $(r=19.8)$ (Lin et al. 2009). Likewise, the brightest knots in these systems also have very high surface brightness, $\sim 23.5$ mag $\operatorname{arcsec}^{-2}$ in $r$, similar to the Clone, and about one magnitude fainter than the 8 o'clock arc. The systems in our sample are thus some of the brightest lensed galaxies known at $z>2$ and will be amenable to detailed studies of their individual properties. 
Combined with our previous results (Allam et al. 2007; Lin et al. 2009; Kubo et al. 2009) the Sloan Bright Arcs Survey has now spectroscopically confirmed 12 strong lensing systems in SDSS DR5 and DR6. There are follow-up observations of all of these systems scheduled for either Cycle 16 Supplemental data or for Cycle 17 of the Hubble Space Telescope. These observations will provide high-quality images useful for detailed lens and source modeling. Additional follow-up observations obtained using WIYN 3.5m telescope at Kitt Peak National Observatory may provide wide-field images of the lensing galaxy that can be used to characterize the galaxy cluster environment. We continue to follow up additional promising lens candidates.

Fermilab is operated by the Fermi Research Alliance, LLC under Contract No. DEAC02-07CH11359 with the United States Department of Energy. These results are based on observations obtained with the SDSS and the Apache Point Observatory 3.5-m telescope, which is owned and operated by the Astrophysical Research Consortium. Funding for the SDSS and SDSS-II has been provided by the Alfred P. Sloan Foundation, the Participating Institutions, the National Science Foundation, the U.S. Dept. of Energy, the National Aeronautics and Space Administration, the Japanese Monbukagakusho, the Max Planck Society, and the Higher Education Funding Council for England. The SDSS Web Site is http://www.sdss.org/.

\section{REFERENCES}

Adelman-McCarthy, J. K., et al. 2007, ApJS, 172, 634

Allam, S. S., et al. 2004, AJ, 127, 1883-1899

Allam, S. S., et al. 2007, ApJ, 662, 51L

Belokurov, V. et al. 2009, Mon. Not. R. Astron. Soc., 392, 104

Bertin, E., \& Arnouts, S. 1996, A\&AS, 117, 393

Bertin, E. 2006, SWarp, http://terapix.iap.fr/IMG/pdf/swarp.pdf. We used SWarp V2.16.5.

Bolton, A., et al. 2008, ApJ, 682, 964

Cabanac, R. A. et al. 2007, A\&A 461, 813

Eisenstein, D. J., et al. 2001, AJ, 122, 2267 
Faure, C., et al. 2008, ApJS, 176, 19

Hainline, K. N., et al. 2009, ApJ, 701, 52

Hansen, S. M. et al. 2005, ApJ, 633, 122

Hennawi, Joseph F. et al. 2008, AJ, 135, 664

Jackson, N. 2008, Mon. Not. R. Astron. Soc., 389, 1311

Kubik, Donna 2007, M.S. Thesis, Northern Illinois University

Kubo, J. M., et al. 2009, ApJL, 696, 61

Lin, H., et al. 2009, ApJ, in press, preprint arXiv:0809.4475

Narayan, R., and Bartelmann, M. 1996, in Formation of Structure in the Universe, ed. A. Dekel and J. P. Ostriker (Cambridge: Cambridge Univ. Press), 1999. (preprint astro-ph/9606001).

Peng, C. Y., et al. 2002, AJ, 124, 266

Shapley, A. E., et al. 2003, ApJ, 588, 65

Smail, I., et al. 2007, ApJ, 654, 33L

Stoughton, C., et al. 2002, AJ, 123, 485

Wen, Z., et al. 2009, Research in Astron. Astrophys., 9, 5

Willis, J.P., et al. 2006, Mon. Not. R. Astron. Soc., 369, 1521

Yee, H. K. C., et al. 1996, AJ, 111, 1783

York, D., et al. 2000, AJ, 120, 1579 

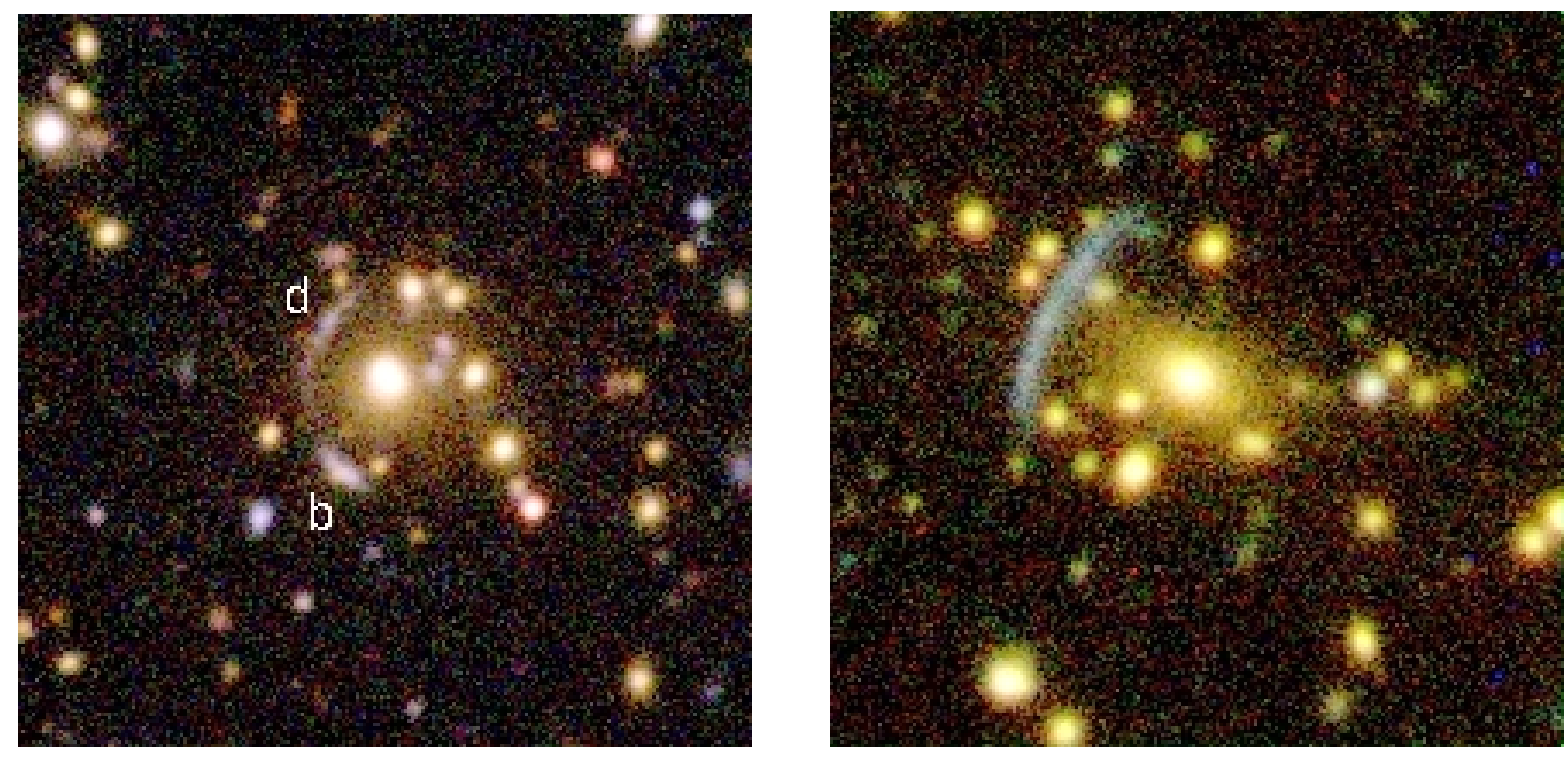

Fig. 1. - Images of SDSS J090122.37+181432.3 (left) and SDSS J134332.85+415503.4 (right). The images are $1^{\prime} \times 1^{\prime}$. North is up. East is to the left. The knots are labeled to distinguish between spectroscopic targets.
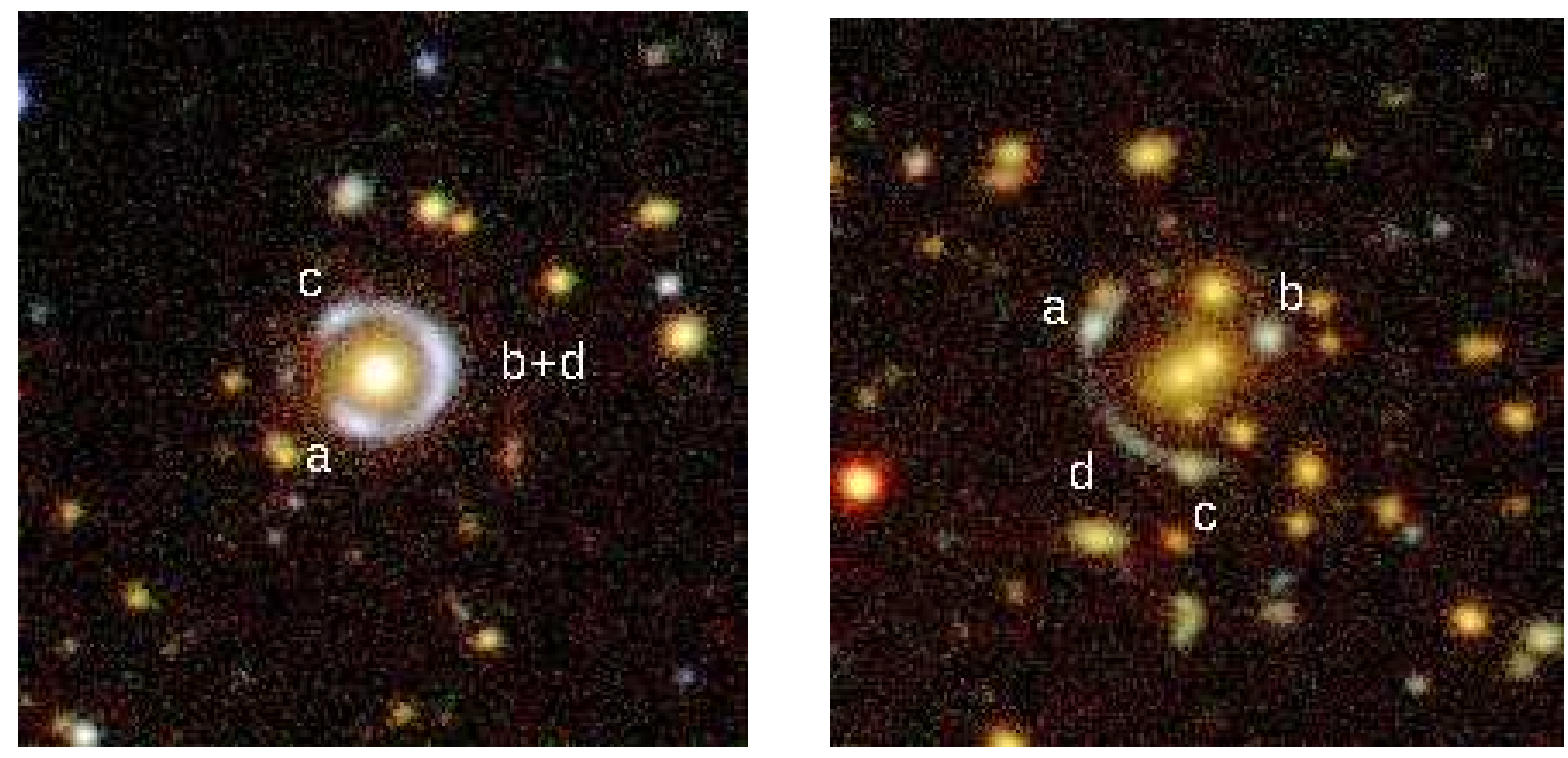

Fig. 2.- Images of SDSS J114833.14+193003.2 (left) and SDSS J090002.79+223403.6 (right). The images are $1^{\prime} \times 1^{\prime}$. North is up. East is to the left. The knots are labeled to distinguish between spectroscopic targets. 


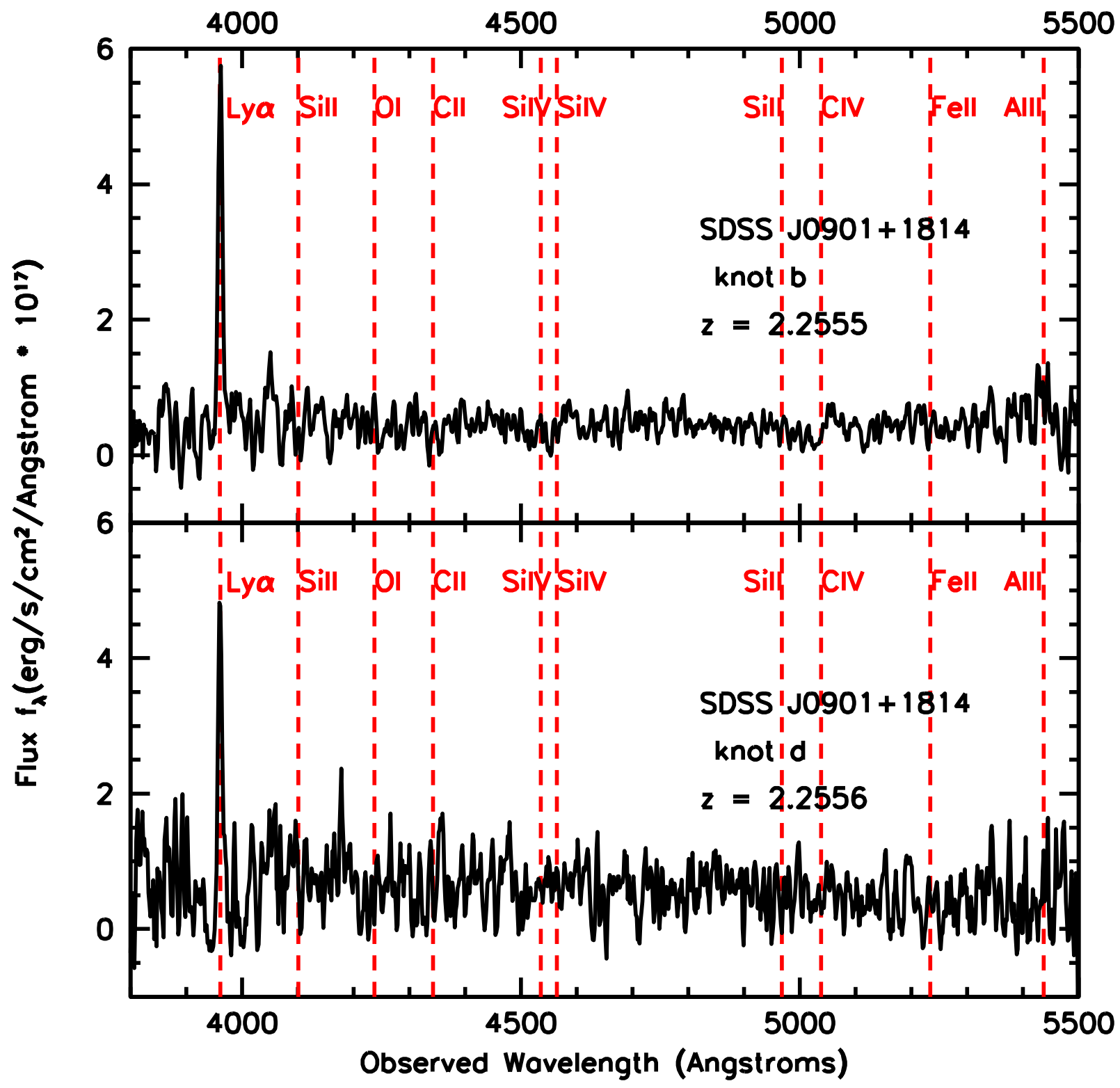

Fig. 3.- Spectra for SDSS J090122.37+181432.3. The upper panel is the spectrum for knot b as shown in Fig. 1 (left). The lower panel is the spectrum for knot d. 


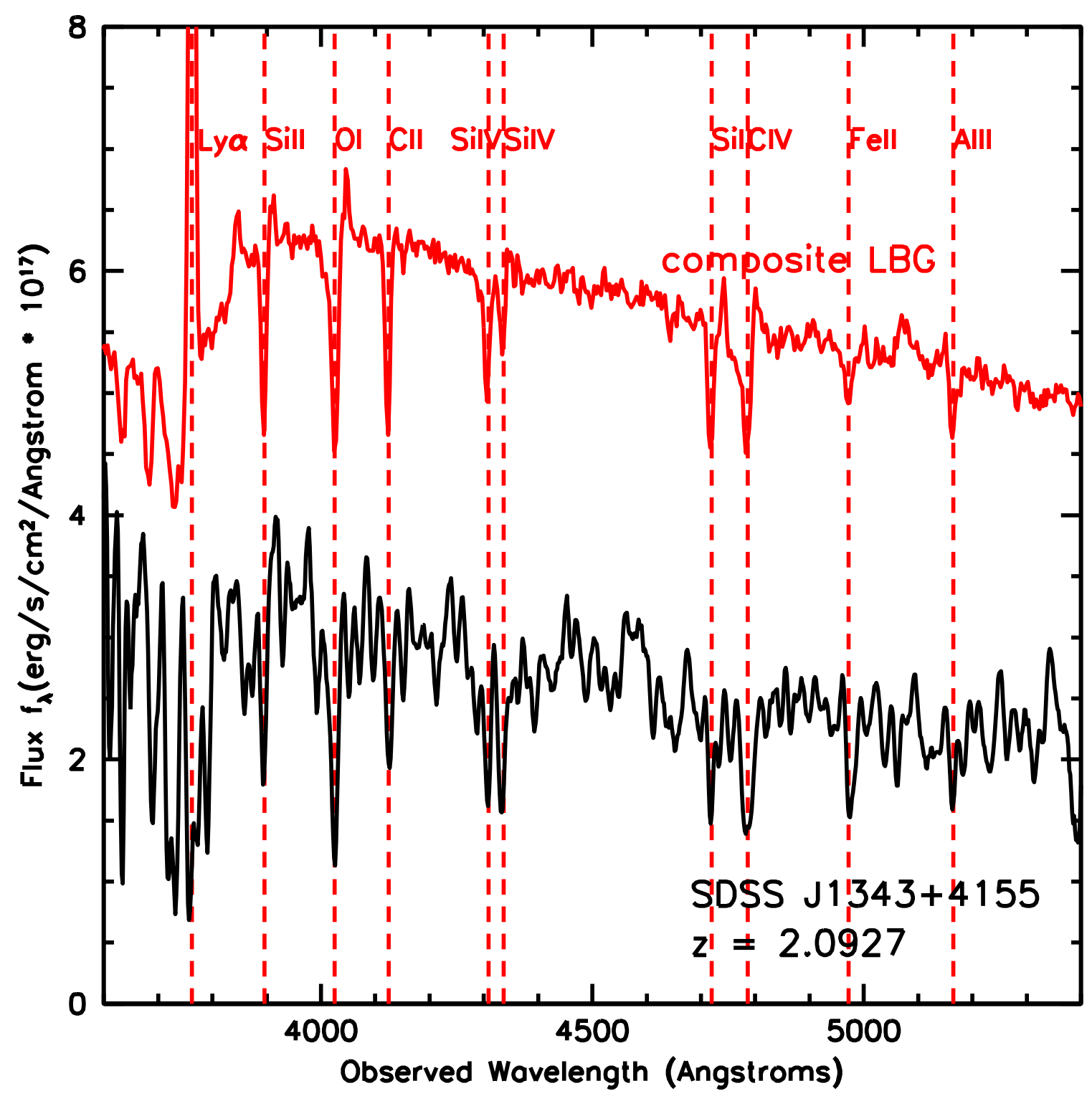

Fig. 4.- Spectrum for SDSS J134332.85+415503.4. The upper spectrum is the composite LBG spectrum of Shapley et al. (2003). The lower spectrum is the arc. We aligned the slit along the arc. 


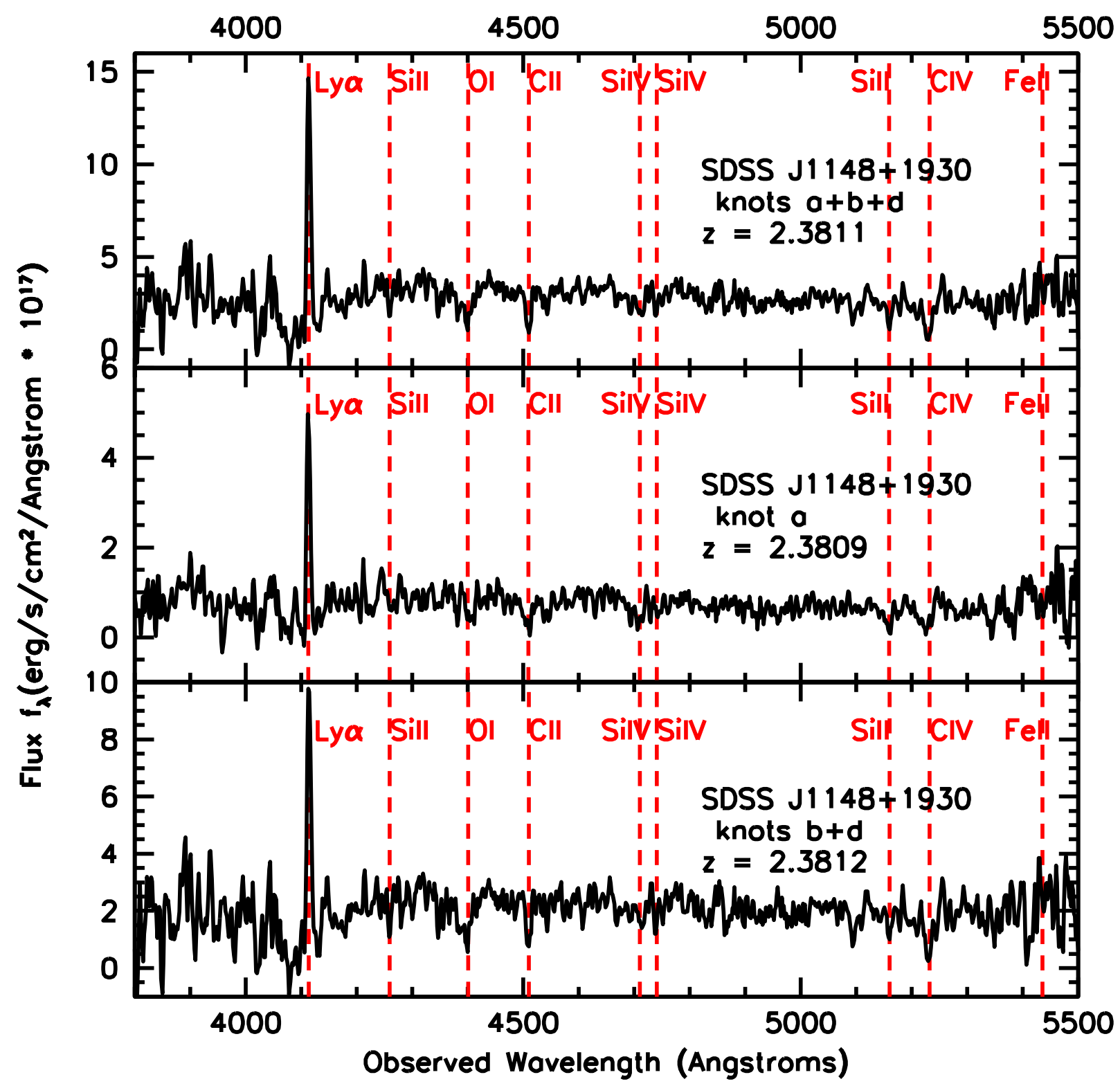

Fig. 5. - Spectra for SDSS J114833.14+193003.2. In the upper panel we show the sum of the spectra for knots a, b, and d as shown in Fig. 2 (left). In the middle panel the slit was oriented so as to cover only knot a. In the lower panel the slit was oriented over knots b and d. 


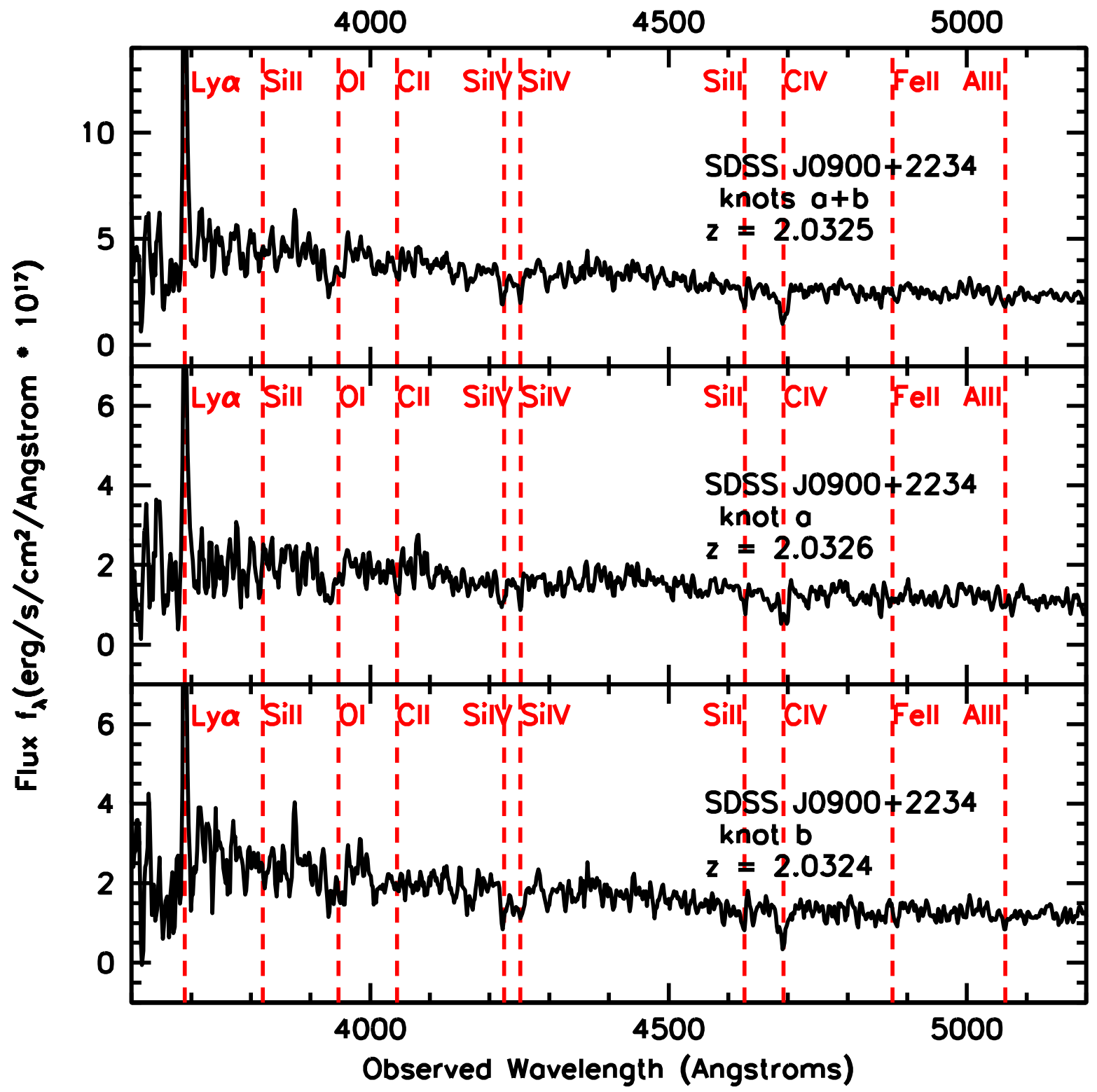

Fig. 6.- Spectra for SDSS J090002.79+223403.6. In the upper panel we show the sum of the spectra from knots a and b. as shown in Fig. 2 (right). In the middle panel we show the spectrum of knot a. In the lower panel we show the spectrum from knot b. 
Table 1: Imaging observation Log. Units of right ascension and declination are degrees.

\begin{tabular}{|c|c|c|c|c|c|}
\hline $\begin{array}{c}\text { System } \\
\text { RA and DEC (J2000) }\end{array}$ & Date & Instrument & Filter & $\begin{array}{l}\text { Exposure } \\
\text { Time }\end{array}$ & $\begin{array}{c}\text { Seeing } \\
g, r, i\end{array}$ \\
\hline $\begin{array}{c}\text { SDSS J090122.37+181432.3 } \\
135.343218 .2423\end{array}$ & 2007 Apr 21 & SPICAM & $g, r, i$ & $3 \times 300$ & $1.0^{\prime \prime}, 0.8^{\prime \prime}, 0.8^{\prime \prime}$ \\
\hline $\begin{array}{c}\text { SDSS J134332.85+415503.4 } \\
205.886941 .9176\end{array}$ & $\begin{array}{l}2008 \text { Jun } 9 \\
2008 \text { Jun } 11\end{array}$ & $\begin{array}{l}\text { SPICAM } \\
\text { SPICAM }\end{array}$ & $\begin{array}{l}g, i \\
g, r\end{array}$ & $\begin{array}{l}2 \times 300 \\
1,3 \times 300\end{array}$ & $1.4^{\prime \prime}, 1.2^{\prime \prime}, 1.4^{\prime \prime}$ \\
\hline $\begin{array}{c}\text { SDSS J114833.14+193003.2 } \\
177.138119 .5008\end{array}$ & 2008 Jan 11 & SPICAM & $g, r, i$ & $3 \times 300$ & $1.2^{\prime \prime}, 1.0^{\prime \prime}, 0.9^{\prime \prime}$ \\
\hline $\begin{array}{c}\text { SDSS J090002.79+223403.6 } \\
135.011622 .5677\end{array}$ & 2006 Nov 22 & SPICAM & $g, r, i$ & $3 \times 300$ & $0.9^{\prime \prime}, 1.0^{\prime \prime}, 1.3^{\prime \prime}$ \\
\hline
\end{tabular}

Table 2: Spectral observation Log.

\begin{tabular}{|c|lccl|}
\hline System & Date & Instrument & Grating & Exposure Time \\
\hline SDSS J090122.37+181432.3 & 2007 Mar 10 & DIS & R400/B300 & $3 \times 900$ \\
& 2008 Mar 11 & DIS & R400/B300 & $1 \times 900$ \\
\hline SDSS J134332.85+415503.4 & 2008 Mar 11 & DIS & R400/B300 & $2 \times 900$ \\
& & & & $1 \times 740$ \\
\hline SDSS J114833.14+193003.2 & 2007 Mar 10 & DIS & R400/B300 & $2 \times 900$ \\
\hline SDSS J090002.79+223403.6 & 2007 Mar 10 & DIS & R400/B300 & $3 \times 900$ \\
\hline
\end{tabular}


Table 3: Strong-lens system properties including the label, right ascension and declination (J2000), and the magnitudes (both isophotal and $3^{\prime \prime}$-diameter circular aperture) of the segments of the source arcs in the $g, r$, and $i$-band filters. Note that for SDSS J134332.85+415503.4, instead of a $3^{\prime \prime}$-diameter circular aperture, we used a curved aperture (a partial elliptical annulus with width $2.5^{\prime \prime}$ and length $21.8^{\prime \prime}$ ) that follows the arc. The "total" magnitudes reported are simply the sum of the magnitudes for the listed arc components. The $r$-band isophotal limits (mag. per arc-seconds ${ }^{2}$ ) are, in order, 25.0, 25.4, 25.5 and 25.2. The statistical uncertainties in the magnitudes are $<0.05$ mag. The units of right ascension and declination are degrees.

\begin{tabular}{|c|c|c|c|ccc|ccc|}
\hline & Arc & & & \multicolumn{3}{|c|}{$3^{\prime \prime}$ mag. } & \multicolumn{3}{|c|}{ isophotal mag. } \\
SDSS System & Segment & RA & DEC & $g$ & $r$ & $i$ & $g$ & $r$ & $i$ \\
\hline J090122.37+181432.3 & b & 135.344265 & 18.240161 & 22.0 & 21.1 & 20.6 & 21.4 & 20.4 & 20.0 \\
& d & 135.344531 & 18.243560 & 22.5 & 21.8 & 21.3 & 21.5 & 20.6 & 20.1 \\
& Total & & & 21.5 & 20.6 & 20.1 & 20.7 & 19.7 & 19.3 \\
\hline J134332.85+415503.4 & NA & & & 20.6 & 20.1 & 19.9 & 20.9 & 20.4 & 20.2 \\
\hline J114833.14+193003.2 & a & 177.138643 & 19.499833 & 21.9 & 21.3 & 21.0 & 21.3 & 20.7 & 20.3 \\
& b+d & 177.136772 & 19.501084 & 21.9 & 21.3 & 21.0 & 20.5 & 19.9 & 19.6 \\
& $\mathrm{c}$ & 177.138971 & 19.502187 & 22.3 & 21.7 & 21.5 & 21.6 & 21.0 & 20.7 \\
& Total & & & 20.8 & 20.2 & 19.9 & 19.8 & 19.2 & 18.9 \\
\hline J090002.79+223403.6 & a & 135.013829 & 22.568782 & 21.2 & 21.2 & 21.2 & 21.0 & 20.9 & 20.9 \\
& b & 135.009554 & 22.568546 & 21.5 & 21.3 & 21.2 & 21.3 & 20.8 & 20.5 \\
& $\mathrm{c}$ & 135.011499 & 22.565599 & 22.3 & 21.6 & 21.3 & 21.9 & 21.3 & 21.0 \\
& $\mathrm{~d}$ & 135.012918 & 22.566221 & 22.7 & 22.4 & 22.2 & 22.3 & 21.9 & 21.7 \\
& Total & & & 20.3 & 20.0 & 19.9 & 20.0 & 19.6 & 19.4 \\
\hline
\end{tabular}

Table 4: Strong-lens system properties including average redshift of the source, the Einstein radius, and enclosed mass of the lens. As noted in the text, we used $h=0.7$.

\begin{tabular}{lcccc}
\hline Lens/Source & $z_{\text {source }}$ & $z_{\text {lens }}$ & $\begin{array}{c}\Theta_{E} \\
\operatorname{arcsec}\end{array}$ & $\begin{array}{c}M\left(<\Theta_{E}\right) \\
\times 10^{12} \mathrm{M}_{\odot}\end{array}$ \\
\hline SDSS J090122.37+181432.3 & 2.2558 & 0.3459 & $7.7 \pm 1.1$ & $9.6 \pm 2.7$ \\
SDSS J134332.85+415503.4 & 2.0927 & 0.4135 & $12.7 \pm 0.4$ & $31.7 \pm 2.0$ \\
SDSS J114833.14+193003.2 & 2.3811 & 0.4457 & $5.0 \pm 0.3$ & $5.1 \pm 0.6$ \\
SDSS J090002.79+223403.6 & 2.0325 & 0.4890 & $7.0 \pm 0.8$ & $11.6 \pm 2.7$ \\
\hline
\end{tabular}




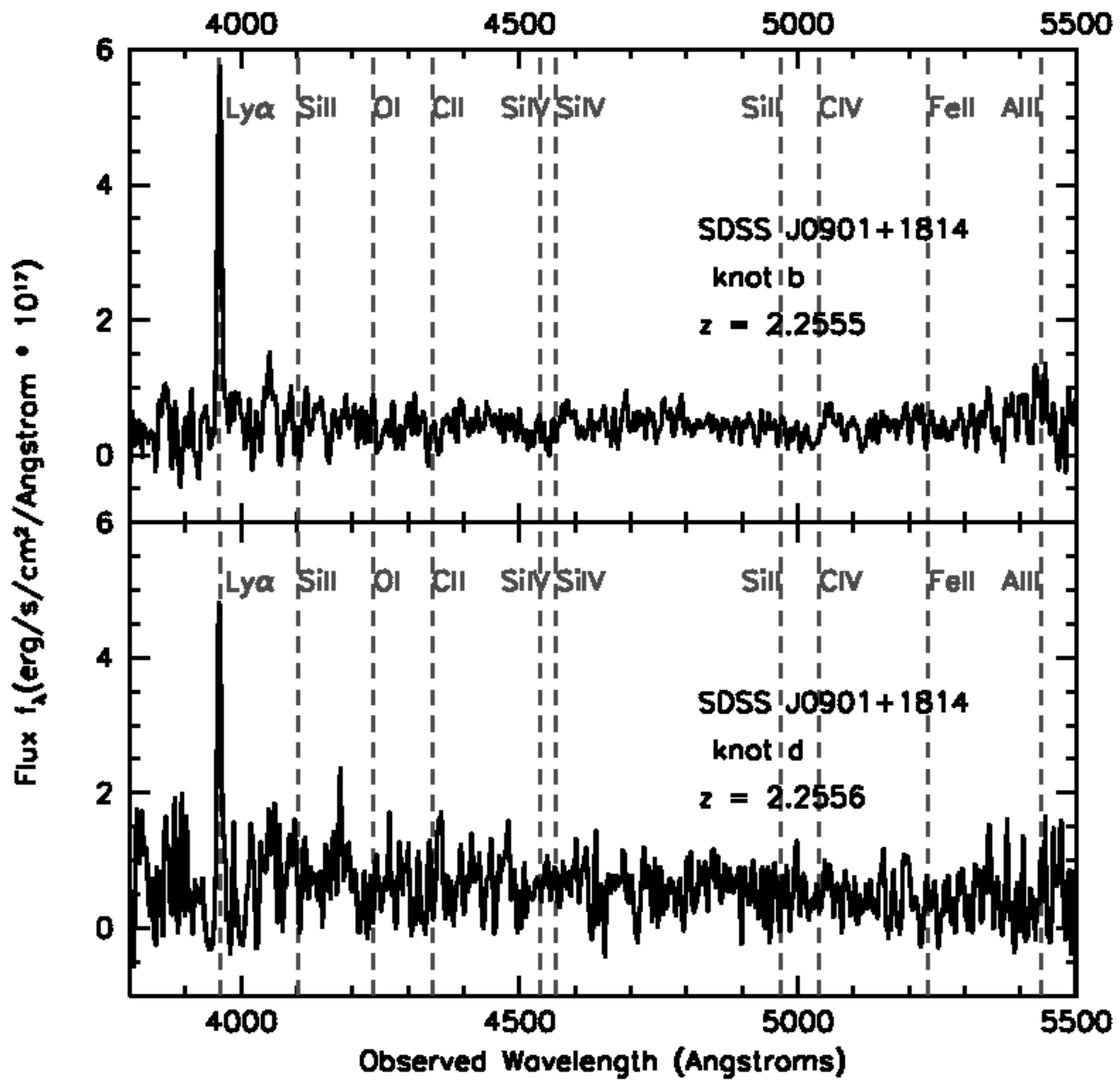




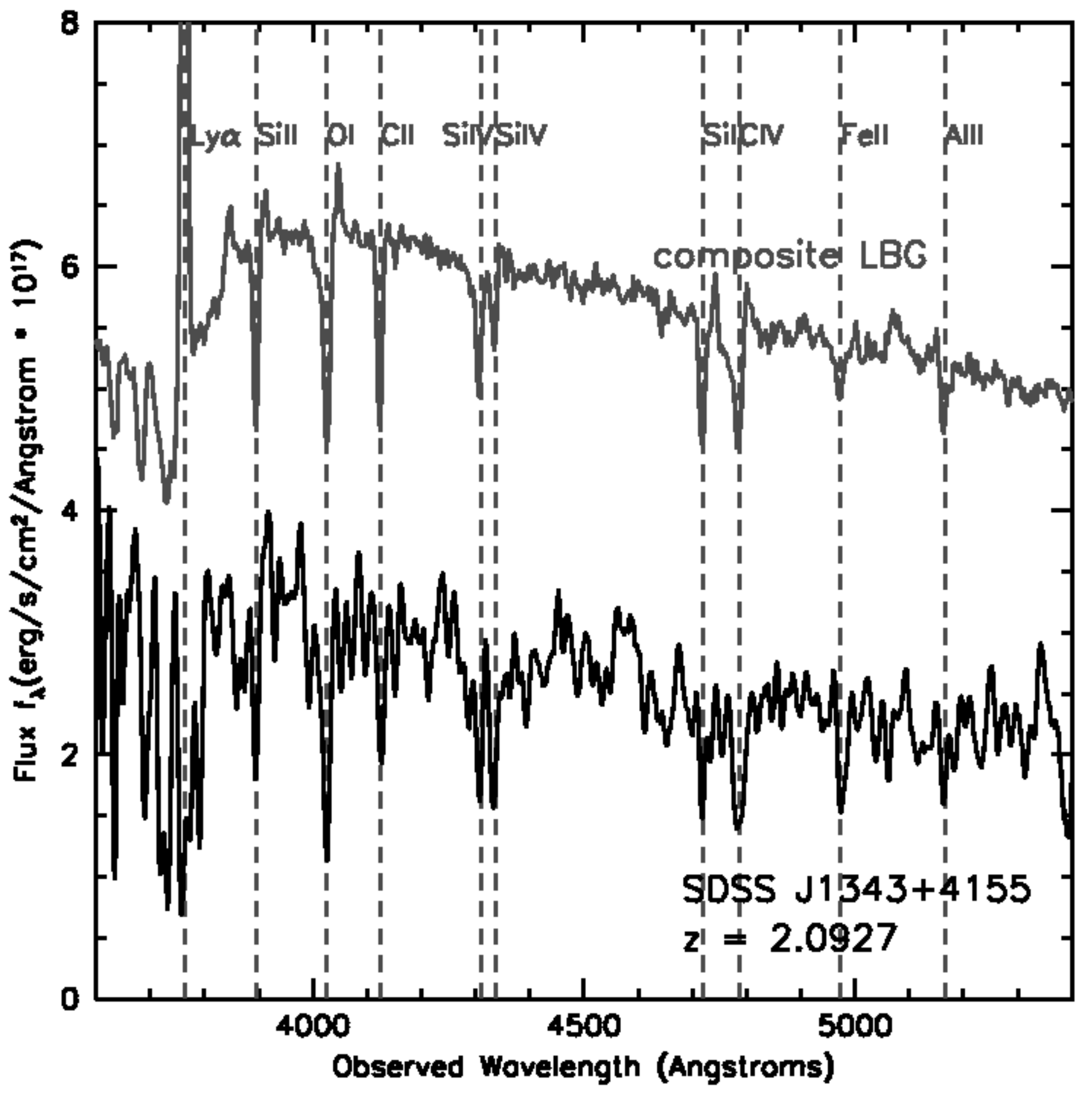




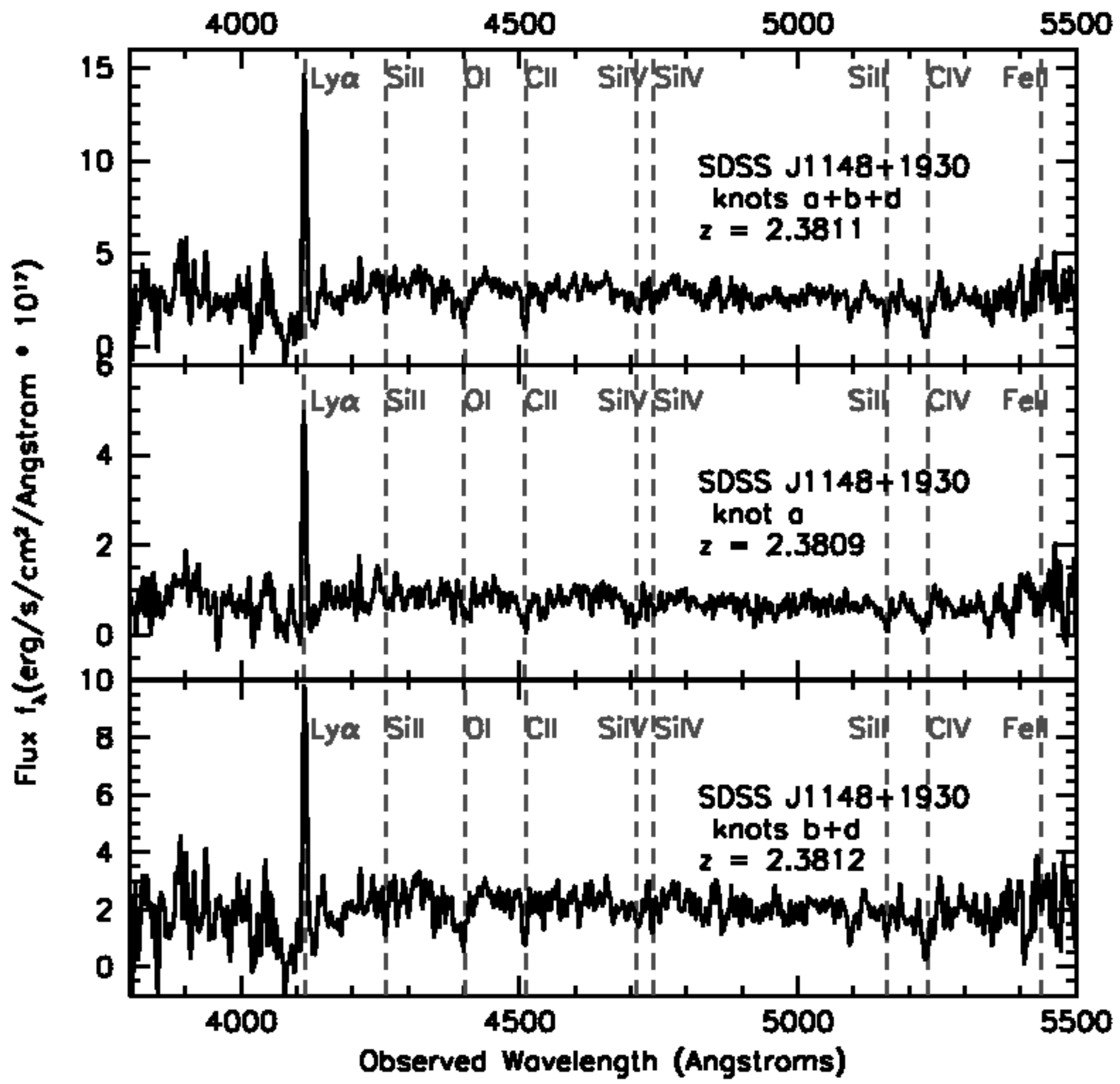




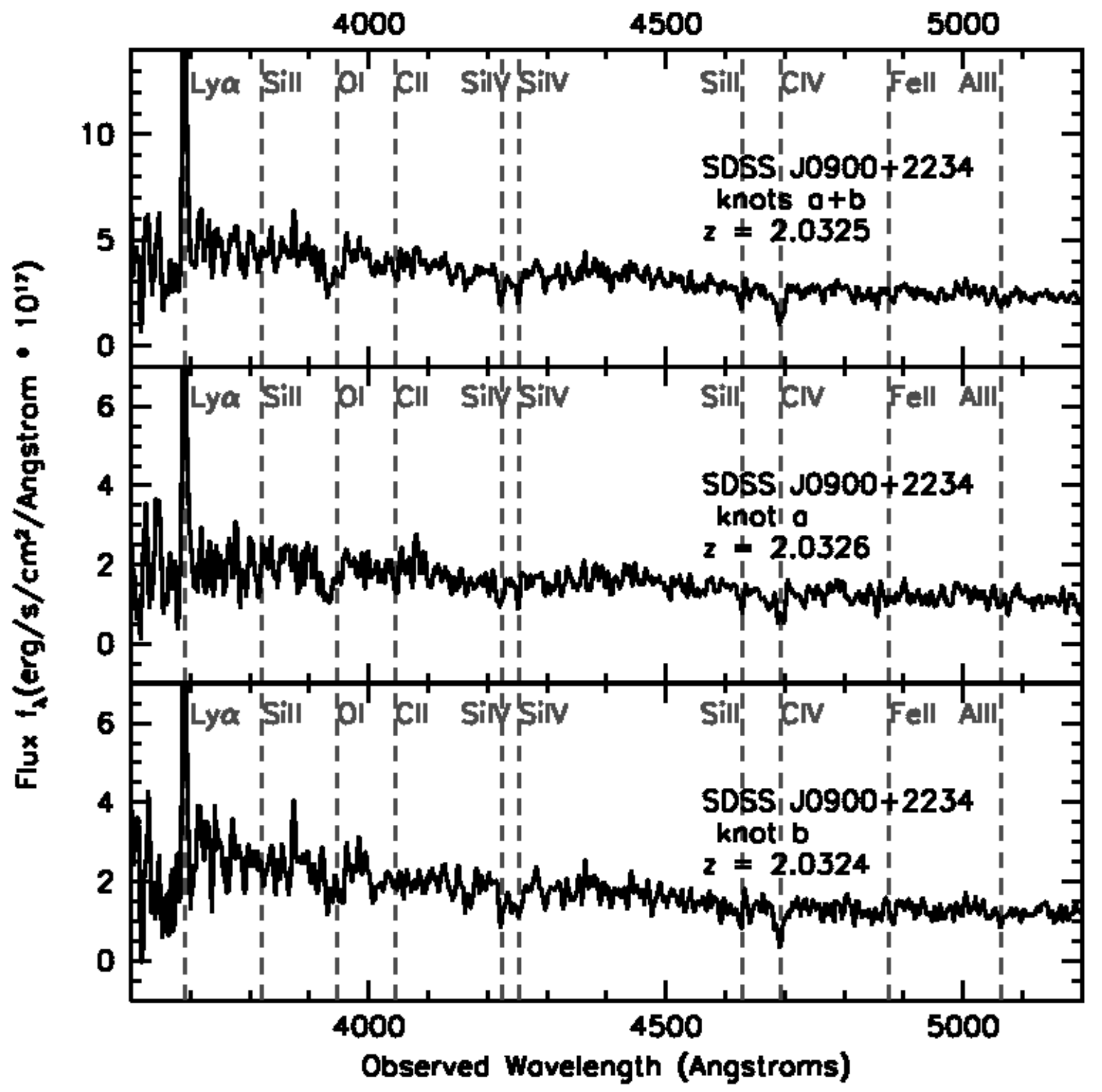

